\section{La Révolution française}

Cahiers de l'Institut d'histoire de la Révolution française

19 | 2021

Appropriation de la Révolution française dans

I'historiographie japonaise

\title{
Le marxisme au Japon, entre émeutes du riz et répression généralisée (1918-1938)
}

Pierre-François Souyri

\section{(2) OpenEdition}

\section{Journals}

Édition électronique

URL : http://journals.openedition.org/lrf/4522

DOI : $10.4000 /$ Irf.4522

ISSN : 2105-2557

Éditeur

IHMC - Institut d'histoire moderne et contemporaine (UMR 8066)

Référence électronique

Pierre-François Souyri, «Le marxisme au Japon, entre émeutes du riz et répression généralisée

(1918-1938) », La Révolution française [En ligne], 19 | 2021, mis en ligne le 01 février 2021, consulté le

04 février 2021. URL : http://journals.openedition.org/lrf/4522 ; DOI : https://doi.org/10.4000/Irf.4522

Ce document a été généré automatiquement le 4 février 2021

(C) La Révolution française 


\title{
Le marxisme au Japon, entre émeutes du riz et répression généralisée (1918-1938)
}

\author{
Pierre-François Souyri
}

\section{NOTE DE L'AUTEUR}

Comme c'est l'usage en Asie orientale, le nom familial précèdera toujours le nom personnel.

$1 \quad$ Les idées socialistes se répandent un peu partout dans le monde dans la seconde moitié du XIX $x^{e}$ siècle, et, à partir de la fin des années 1890, le Japon devient, à son tour, l'un des terreaux sur lequel elles peuvent se développer. Le Manifeste du Parti Communiste est traduit en japonais en 1904 par deux dirigeants socialistes. L'un, Kôtoku Shûsui (1871-1911), passe finalement à l'anarchisme vers 1907 et est exécuté en 1911, accusé avec une quinzaine de camarades - sans preuves et dans le cadre d'un procès monté de toutes pièces - d'avoir voulu attenter à la personne de l'empereur'. L'autre, Sakai Toshihiko (1871-1933), devient alors le principal représentant du socialisme dans l'archipel, puis le président du premier Parti Communiste japonais (créé en juillet 1922, dissous en mars 1924). Kôtoku Shûsui avait livré dès 1901 une analyse originale de l'impérialisme ${ }^{2}$. En 1907, le socialiste Yamakawa Hitoshi (1880-1957) publia une introduction aux premières parties du Capital ${ }^{3}$.

2 Après 1911 et le procès contre les anarchistes, la répression, pour ne pas dire la terreur, s'abat sur le mouvement syndical tout comme sur le mouvement socialiste. Ce dernier est pratiquement décapité et entre dans ce qu'on désigne en japonais comme «l'ère d'hiver »: arrestations, intimidations, les militants sont devenus les cibles de la répression policière et, de fait, inaudibles. Critiques de l'insuffisance de la démocratie et de la timidité du mouvement syndical, les socialistes se sentent néanmoins encouragés par la tournure que prennent les événements en Russie en 1917, mais c'est 
surtout après les grandes émeutes du riz (été 1918) que leur activité se fait plus visible. $\mathrm{Au}$ bout du compte, l'aggravation sensible des divisions sociales pendant les années 1910, la distribution inégale de la richesse, les difficultés économiques combinées à l'impéritie des classes possédantes, ont poussé le peuple dans la rue et la propagande socialiste peut de nouveau faire mouche. Il s'agit là d'une crise politique, sociale, économique de grande ampleur qui, d'une certaine manière, contribue à « délivrer une société bloquée" par un couvercle de plomb, celui de la répression contre le mouvement social. Les anciens dirigeants du mouvement jouent alors le rôle de cadres, faisant le pont entre l'ancienne génération de socialistes des années 1900-10 et une nouvelle génération qui surgit vers 1920. Pour eux, les émeutes du riz marquent un tournant dans la montée de la conscience de classe populaire ${ }^{4}$.

3 À partir de 1919 et pendant plusieurs années, conflits, manifestations de rue et grèves se multiplient. La colère ouvrière contre un système industriel quasi-carcéral est vive. C'est à cette époque que se met en place au Japon une culture ouvrière du conflit, avec ses chants de lutte, ses manifestations interdites le $1^{\text {er }}$ Mai, et les démonstrations de joie organisées à la sortie de prison des militants arrêtés.

4 Vers 1919-20, Sakai Toshihiko et ses camarades ont pour objectif de rassembler les divers courants ouvriers et étudiants apparus depuis 1918 pour les regrouper en une Fédération socialiste japonaise dont l'objectif serait «la destruction complète du système capitaliste", l'instauration d'une société libre, égalitaire, pacifique et fraternelle, par le moyen de la «lutte des classes». Sakai Toshihiko et Yamakawa Hitoshi incarnent alors ce qu'on peut appeler un courant se réclamant de l'héritage intellectuel de Marx. Ils sont rejoints par des syndicalistes radicaux, des écrivains et de jeunes étudiants. Il s'agit de créer un parti socialiste combatif, radical et unifié. Lors du congrès officiel de fondation de la Fédération en décembre 1920, la nouvelle organisation revendique trois mille membres, mais le parti est dissout par la police en mai 1921. Cependant le phénomène vraiment nouveau, c'est que les idées socialistes se répandent pour la première fois de manière substantielle dans le monde intellectuel comme dans la classe ouvrière et que, partout, elles suscitent un intérêt réel. Déjà, l'ouvrage de l'économiste Kawakami Hajime, Récits de la pauvreté (Binbô monogatari), paru en 1917, avait connu un fort succès. L'auteur y montre comment le capitalisme, au lieu d'améliorer les conditions de vie des classes prolétaires, ne faisait que les aggraver et il appelle à la subversion du système. Takabatake Motoyuki, éditeur d'une revue Kokka shakai shugi (Socialisme d'État) accueille de son côté avec enthousiasme la révolution bolchevique et traduit pour la première fois le Capital de Karl Marx en japonais (1920-22)

5 Le rapprochement entre le parti bolchevik russe et les socialistes radicaux japonais s'opère en effet, bien que lentement. À vrai dire, la position géographique du Japon, combinée au barrage de la langue, joue dans le sens d'un isolement relatif du mouvement socialiste dans l'archipel. Avant 1917, on ignorait tout de l'évolution de la social-démocratie russe et Lénine était inconnu. En 1917, Sakai Toshihiko le considère comme une sorte d'anarchiste et Yamakawa Hitoshi pense que les bolcheviks sont des anarcho-syndicalistes partisans de l'action directe ${ }^{6}$. Il faut attendre 1921 pour que paraissent les premières traductions en japonais des ouvrages de Lénine et que le bolchevisme apparaisse comme une rupture dans ce que l'on savait au Japon de l'histoire du socialisme. L'Impérialisme, stade suprême du capitalisme de Lénine (1915) est traduit en 1924. C'est d'ailleurs la traduction en japonais vers le milieu des années 1920 
de l'ABC du communisme (1919) de Boukharine qui constitue alors l'ouvrage le plus répandu au Japon sur le bolchevisme.

Du côté des socialistes radicaux, qualifiés d'« extrémistes » dans les médias, la figure de Yamakawa Hitoshi s'impose peu à peu comme celle d'un leader théorique à côté de Sakai Toshihiko, le leader historique. L'ampleur des luttes ouvrières, combinée au prestige de la révolution russe, donne naissance à une pensée marxiste japonaise de plus en plus radicale. Le contexte a en effet évolué et, avec la multiplication des conflits du travail, les socialistes radicaux tentés par le communisme changent complètement de stratégie. C'est Yamakawa Hitoshi qui se fait le porte-parole et le leader des groupes «bolcheviks» dans un essai publié l'été 1922 et intitulé Changement de cap pour le mouvement prolétarien ${ }^{7}$. Yamakawa y rejette définitivement toute pensée de type millénariste - ce qu'en Occident, on qualifierait de socialisme utopique - pour se tourner vers le marxisme et s'en prend aux radicaux mus par leur indignation morale, leur critique superficielle du système capitaliste jugé corrupteur, c'est-à-dire critique les formes prises par le premier mouvement socialiste des années 1900, alors qu'il était sous l'influence intellectuelle de Kôtoku Shûsui. Ce socialisme-là se situait dans une logique "asiatique ", c'est-à-dire dans l'idée très confucéenne que l'harmonie sociale est le produit d'une forme de vertu et que le chaos est le fruit de l'excès. Égoïsme et individualisme conduisent, sous prétexte de liberté, à créer de la pauvreté. C'est donc au nom d'autres principes, plus moraux, que l'ordre peut être rétabli. En fin de compte, ce n'est pas l'économie elle-même mais la vertu, mise en œuvre par l'État, qui peut combattre les désordres de l'économie. Contre cette forme de pensée désormais dépassée, Yamakawa explique que le nouveau mouvement socialiste doit désormais être rigoureux et intelligent dans ses choix politiques ${ }^{8}$.

7 Yamakawa repère ce qu'il conçoit comme une contradiction intrinsèque du nouveau mouvement social : à critiquer de trop le syndicalisme modéré, on «se coupe des masses " et, à trop suivre les revendications populaires, on «perd de vue l'objectif final». Le but ultime du mouvement est la destruction du capitalisme et les revendications salariales ouvrières ne le mettront pas en péril, explique-t-il. Mais c'est pourtant dans la lutte au quotidien que doit se forger la conscience du but ultime. Il faut donc accompagner la classe ouvrière dans ses revendications; c'est le rôle de l'avant-garde que de « conduire » les masses populaires vers une conscience claire de la nécessité de la révolution.

8 Ce discours radical, nouveau au Japon, est, on le comprend, très influencé par Lénine. Pour Yamakawa, la révolution ne sera pas possible sans le rôle «éclairé » de l'avantgarde formée par les cadres «bolcheviks». Pourtant, Yamakawa n'est pas totalement convaincu de la nécessité indiscutable d'une avant-garde éclairant un prolétariat qui resterait inerte sans son intervention. Il explique dans le même texte que la conscience de classe ne peut non plus se comprendre comme le simple fruit d'une pratique de gens érudits et isolés. Pour surmonter cette contradiction, il faut, selon lui, peser sur le cours jugé trop modéré des syndicats et développer l'agitation politique en vue de l'obtention du suffrage universel, qui permettra de faire élire des représentants prolétariens exprimant un point de vue de classe au parlement. Et, en même temps, il faut participer au débat politique général en intervenant régulièrement dans des revues réformistes comme Kaizô $\hat{0}^{9}$ et éduquer la classe ouvrière: les premiers communistes font en effet un travail de vulgarisation non négligeable en direction des travailleurs à travers l'organisation de cours du soir et d'écoles de formation. 
9 À côté des leaders au Japon, d'autres militants japonais agissent aussi à l'étranger. C'est le cas de Katayama Sen, qui prend langue aux USA entre 1914 et 1917 avec les exilés russes, y compris Boukharine et Trotsky, et adhère au PC américain en 1919. En 1921, Katayama s'installe à Moscou, où il joue un rôle central au Komintern pour la mise en place d'une politique communiste japonaise. C'est aussi le cas de Tokuda Kyûichi (le futur secrétaire général du parti en 1945), séjournant également en Russie et qui repart en 1922 au Japon avec des instructions et des fonds pour y créer un parti. Finalement le 15 juillet 1922, le parti communiste japonais est fondé clandestinement et élit Sakai Toshihiko comme président. La nouvelle organisation ne comptait guère qu'une cinquantaine de membres, semble-t-il. La fondation du PCJ fut tellement clandestine que, en fait, on ne sait pas très bien quand elle eut réellement lieu! Certains évoquent une date plus précoce, mars ou avril $1922^{10}$. Le groupe est néanmoins reconnu en novembre comme la section japonaise du Komintern, mais c'est un groupe dispersé et virtuel que reconnaît là l'Internationale communiste.

10 À partir de 1921-22, le mouvement socialiste est divisé en trois ou quatre entités : les socialistes qui ne rallient pas la cause bolchevik et qui sont divisés eux-mêmes en "socialistes de gauche» et sociaux-démocrates modérés; les communistes; et les anarchistes. La répression les touche tous de manière systématique. Censure, dissolutions, arrestations plus ou moins longues, condamnations rendent la vie des militants difficile ${ }^{11} \ldots$ Le mouvement socialiste éclate en plusieurs groupuscules, dont les différences sont souvent minimes : il s'agit souvent de groupes locaux dispersés par la répression. Cet ensemble de groupes constitue finalement une mouvance qui est loin d'être négligeable, celle dite des partis prolétariens.

Ceux-ci se regroupent finalement fin 1925 sous la direction d'Ôyama Ikuo, un socialiste de gauche, professeur à l'université Waseda, à la faculté d'économie. Ils refusent l'adhésion au Komintern et fondent le Parti paysan et ouvrier. Regroupant plusieurs centaines de congressistes, le parti est dissous après une demi-heure d'existence [sic !]. Il se reconstitue quelques semaines plus tard au début de 1926 sous le nom de Parti ouvrier et paysan [sic!], dissous de nouveau en avril $1928^{12}$. Cette fois, il explose en de multiples tendances, plus ou moins radicales, toujours éphémères. Aux élections de 1928, les premières élections au suffrage universel masculin, les partis prolétariens, malgré des intimidations innombrables, obtiennent vingt-huit élus - la plupart issus de circonscriptions ouvrières - et forment un groupe parlementaire non négligeable.

De son côté, le parti communiste clandestin se retrouve vite dans une situation critique. Rassemblés autour d'un noyau de militants socialistes radicalisés mais " coupés des masses", les communistes sont confrontés à un dilemme: survivre telle une secte ou se dissoudre «dans le mouvement de masse » où les idées communistes se développent rapidement, notamment dans les syndicats. En fait, les dirigeants du nouveau parti sont tous des «anciens» du mouvement socialiste, influencés par des idéologies diverses : social-démocratie, anarcho-syndicalisme... Une dose de taoïsme et de bon sens populaire ne les quitte pas. La plupart sont d'anciens autodidactes qui, révoltés par l'injustice et la corruption, se sont radicalisés ${ }^{13}$. Contrairement au Komintern qui les presse d'agir et de s'organiser, ils pensent que, dans l'atmosphère de répression qui caractérise l'année 1923, notamment après le grand séisme du Kantô et les pogroms anti-coréens, il est inutile de brûler les étapes et il est préférable d'attendre que "les masses mûrissent ${ }^{14}$ ». Le parti est officiellement dissous en mars 1924, contre l'avis de Moscou. Pour les dirigeants du premier parti, la constitution 
d'une secte isolée n'a aucun sens, d'autant que les tendances de plus en plus autoritaires émanant de Moscou commencent à les rebuter. Ils font un constat pourtant : les idées communistes sont en progression partout, mais pas le parti. À Kyôto par exemple, le professeur d'économie Kawakami Hajime (1879-1946) fait lire Marx à ses étudiants, édite sa propre revue et attire des jeunes à la cause sans pour autant être membre officiel. Pour Yamakawa, il faut développer une « ligne de masse ", c'est à dire constituer un front uni avec « la bourgeoisie » japonaise et avec les partis prolétariens contre les forces réactionnaires. Le repli sectaire comporte d'ailleurs un autre risque : celui d'exposer inutilement des militants isolés à la répression.

\section{L'essor du marxisme}

13 Comme théorie du changement et de l'explication du monde, le marxisme attire de nombreux intellectuels, notamment parmi les jeunes. Créée en décembre 1918 comme association d'enseignants et d'étudiants de l'université impériale de Tokyo, la shinjinkai, qui professait le libéralisme politique, devient rapidement un foyer de diffusion des idées marxistes et le noyau de la future Fédération des étudiants, qui regroupe un grand nombre d'étudiants issus de différentes institutions académiques, très influencée par ceux se réclamant du marxisme. À partir des années 1920 émergent par ailleurs des figures d'intellectuels marxistes, comme Yamakawa Hitoshi ou Kawakami Hajime, qui participent au débat général d'opinions, dans la presse par exemple, en tant qu'essayistes ou chroniqueurs. Les mouvements sociaux de cette époque, le mouvement ouvrier bien sûr mais aussi les mouvements paysans ${ }^{15}$, le mouvement de libération des parias ${ }^{16}$, les burakumin, le mouvement étudiant, furent considérablement influencés par le marxisme, notamment entre le milieu des années 1920 et les premières années 1930. Dans la seconde moitié des années 1920, le marxisme commence, au Japon, à devenir un vrai phénomène intellectuel ; plusieurs médias évoquent alors l'invasion des ouvrages marxistes, des analyses marxistes, des traductions d'auteurs marxistes européens, à commencer par Marx et Lénine, sur les rayons des librairies et des bibliothèques, dans les articles de revues ${ }^{17}$. On parle des jeunes étudiants'es comme des « Marx boys » et des « Engels girls ». Cet intérêt pour le marxisme au Japon, véritable phénomène culturel, est peut-être sans commune mesure avec ce qui peut se passer ailleurs.

Les premiers socialistes étaient des journalistes et des agitateurs, rarement des universitaires, qui avaient délibérément interrompu leurs études pour embrasser une cause,. Le succès social leur était indifférent. Il n'en va pas de même de la génération de 1920, qui sort des meilleures universités du pays, voire y enseigne, ou y enseignera. Les plus belles carrières sont promises à ces étudiants brillants, qui sont persuadés que le système capitaliste est au bord de l'effondrement. Ils occupent parfois des chaires universitaires, créées en grand nombre à cette époque, souvent au retour d'un séjour d'études en Allemagne. Mais, en adoptant le marxisme, ils offrent à celui-ci une respectabilité morale et une influence intellectuelle.

15 Parce que le marxisme s'intéresse, d'un point de vue neuf pour l'époque, à la question politique ou sociale ou à l'économie, parce que le marxisme est alors une pensée en construction, ouverte et innovante, susceptible de provoquer des débats, il suscite un intérêt très fort. Philosophes, autour de Tosaka Jun (1900-1945), artistes et écrivains, autour de la Ligue des artistes prolétariens, étudiants des universités, regroupés en divers cercles de lecture, se passionnent pour le mouvement communiste, qu'ils 
adhèrent ou non au PCJ ou aux partis prolétariens. Les marxistes sont le premier courant politique au Japon à avoir, auprès de ceux qui défendent leur cause, "une exigence scientifique», qu'on se représente souvent comme une exigence « universelle » et «moderne », et, dans les faits, le mouvement intellectuel marxiste japonais atteint un niveau certain de raffinement théorique, souvent inconnu ailleurs, en tous cas dans le reste de l'Asie.

Dans ses travaux publiés à la fin des années 1920, Kawakami Hajime montre que la croissance du capitalisme au Japon ne provoque qu'une dégradation des conditions de vie des travailleurs, si ceux-ci se laissent exploiter en silence sans réagir. Mais, pire encore, le capitalisme désintègre les valeurs japonaises traditionnelles - l'entraide, la solidarité, le sens de la collectivité - par sa poursuite implacable du profit, qui favorise la montée de comportements individualistes et égoïstes. D'une certaine façon, le marxisme radical réintroduit des valeurs traditionnelles, comme celle du collectivisme, que l'individualisme capitaliste « occidental » est en train de détruire. Il offre un moyen de maintenir et développer les valeurs traditionnelles sans se replier sur le passé. En se faisant l'écho de certaines inquiétudes qui puisent dans le passé national, les marxistes cherchent à fournir aussi une alternative au débat désormais classique entre partisans de l'occidentalisation et tenants de la défense des valeurs nationales. Cette capacité du marxisme japonais à s'intégrer dans un discours national aurait pu faire sa force si ces tentatives n'avaient été brisées avant-guerre par le sectarisme du Komintern, d'une part, et la répression, d'autre part.

17 L'historien des idées Maruyama Masao écrivait en 1968 que l'influence du marxisme d'avant-guerre était bien sûr liée à l'influence politique du Komintern et du Parti communiste japonais, mais qu'il s'agissait surtout d'un mouvement intellectuel dont la dimension avait été considérable ${ }^{18}$. En fait, ce «mouvement intellectuel» fut un mouvement théorique, mais aussi un mode général de pensée et de comportement qui avait des implications diverses dans le style de vie des gens qui s'en réclamaient : étude, militantisme, clandestinité, arrestation, prison etc... Devenir marxiste dans le Japon des années 1925-35, c'est-à-dire à l'époque de son apogée, était plus qu'un choix intellectuel et politique : cela pouvait avoir des conséquences sur sa propre vie, voire celle de ses proches.

\section{Le marxisme en débat : Fukumoto contre Yamakawa}

18 Le PCJ se reconstitue néanmoins, bien que de manière clandestine, en 1926. Certains, d'ailleurs, évoquent cette refondation comme le nouveau départ d'un parti plus encadré et soumis à Moscou. Sakai, Yamakawa et les "socialistes historiques", partisans du front commun avec les autres partis prolétariens, se sentent alors marginalisés et doivent céder la place à des militants plus radicaux regroupés derrière Fukumoto Kazuo (1894-1983), qui, lui-même, se fait le chantre du sectarisme au nom de la pureté idéologique et de la "division avant la réunification ", courant qu'on se met à désigner en japonais sous le terme de « fukumoto-isme ».

Selon Fukumoto, il faut rompre avec l'amateurisme des anciens socialistes passés au communisme : cette génération de Meiji n'est pas au fait de l'internationalisme tel qu'il se développe en Europe et reste trop influencée par le confucianisme, le christianisme, le culte de l'État chez certains, l'économisme chez d'autres, c'est-à-dire par le socialisme utopique dans toutes ses variantes. La reconstitution du parti doit se refaire, 
portée par les cadres formés à Moscou, sous la conduite théorique de Fukumoto. Celuici veut débarrasser le mouvement de ses tendances syncrétiques et de son "confusionnisme", fruit de la période précédente, pour écrire une nouvelle page blanche du communisme fondée sur une véritable compréhension du marxisme à la mode léniniste. Dans plusieurs essais publiés en 1925-26, il accuse Yamakawa Hitoshi ou Kawakami Hajime de tenir des positions étrangères au marxisme, invite les militants à se libérer de leur influence et les exhorte à approfondir leur réflexion théorique, en particulier le maniement de la dialectique.

À la différence des anciens leaders socialistes devenus communistes, Fukumoto Kazuo est non seulement un "jeune", mais aussi un diplômé de la prestigieuse faculté de droit de l'université de Tôkyô. Envoyé comme boursier du gouvernement dans l'Allemagne de la république de Weimar, il s'était confronté aux classiques du marxisme allemand, mais avait lu aussi les œuvres de Rosa Luxembourg, Karl Korsch et Georg Lukacs, qu'il contribue à faire connaître au Japon. Il fascine les étudiants des universités dans lesquelles il donne des conférences à son retour et parvient à attirer autour de lui une nouvelle génération de jeunes cadres issus des meilleures universités. Esprit vif, doué pour la polémique il en use "à la communiste", sans se soucier du nécessaire respect dû aux anciens. Le Changement de cap de Yamakawa en 1922 et la dissolution du parti en 1924 étaient dus, selon lui, au fait que les premiers dirigeants du parti ne sont pas vraiment " léninistes ", incarnent " des tendances économistes » qui collent aux revendications ouvrières, mais perdent de vue l'objectif final. Lui est un farouche partisan d'une avant-garde idéologiquement consciente, condition nécessaire à l'émergence d'un vrai mouvement de classe. L'idée du front uni promue par Yamakawa, dans lequel peuvent se reconnaître non seulement la classe ouvrière, mais aussi les petits paysans, les parias (burakumin), les colonisés et les fractions les plus progressistes de la bourgeoisie, est une absurdité. Fukumoto insiste sur la pureté idéologique nécessaire de l'avant-garde et dénonce la passivité de ses adversaires. Son insistance sur la pureté de la ligne et l'invariance des principes n'est pas sans rappeler d'ailleurs les positions d'Amedeo Bordiga en Italie ${ }^{19}$.

21 De son côté, Yamakawa critique les partisans de l'abstraction et les tenants du sectarisme. En 1926, Yamakawa refuse de participer à ce parti communiste reconstitué sous influence fukumoto-iste. Ce qui marche en Russie ne marche pas nécessairement au Japon, déclare-t-il en substance. L'année suivante, Yamakawa et son groupe rejoignent le Parti ouvrier et paysan, animé par des socialistes de gauche, hostiles au communisme tel que l'incarne désormais l'Union soviétique.

22 Le débat qui oppose Yamakawa et Fukumoto reflète évidemment un conflit de génération politique et une lutte de pouvoir, mais pas seulement. D'abord, le contrôle de l'appareil du parti qui est souvent l'objet des luttes politiques entre tendances dans le mouvement communiste, n'a pas lieu d'être ici, puisqu'il n'existe vers 1925 pas de parti, donc pas d'appareil. Yamakawa et Fukumoto se déchirent, mais le Komintern les rejette finalement l'un comme l'autre. Yamakawa est considéré comme un liquidateur dès 1924-25 et Fukumoto comme un gauchiste en 1927, depuis qu'il s'est permis de critiquer Lénine lui-même. Le fukumoto-isme ne convient pas à Moscou : il s'agit d'une tendance trop sectaire et, surtout, insuffisamment soumise au Komintern et à l'URSS. Convoqué à Moscou, critiqué par Boukharine, Fukumoto est désavoué20.

Dès 1927, le parti communiste clandestin est en effet peu à peu repris en main par Moscou où, entre-temps, Staline a consolidé son influence sur l'État soviétique. Toute 
tentative de mettre en place une organisation politique nationale défendant ses propres objectifs et ayant sa propre stratégie sera désormais contrecarrée avec succès par le Komintern, qui multiplie, à la fin des années 20, des stages à Moscou pour les dirigeants du parti. Cette reprise en main reste cependant illusoire, car le Parti communiste japonais, affaibli par les arrestations massives, ne subsiste que comme organisation minoritaire, étroite et repliée sur elle-même : jusqu'à l'après-guerre, le PCJ se révèle incapable d'offrir une continuité en terme de leadership, d'organisation et de stratégie $^{21}$. En fait, il existe, dans le Japon des années 1925-35, une sorte de décalage grandissant entre un Parti communiste ultra-minoritaire, clandestin, placé sous tutelle par Moscou, et le marxisme japonais qui, lui, se construit comme une forme de pensée dynamique et productive.

Le marxisme de Yamakawa est en effet un marxisme vivant, pas complètement passé par la moulinette idéologique du marxisme-léninisme. Il y a, chez Yamakawa et, plus tard, chez les tenants de la tendance dite rônô (Ouvriers et paysans), une sorte de réaction anti soviétique à l'idée d'une ligne qui serait définie depuis l'extérieur. On peut même émettre l'hypothèse d'une rupture dans le marxisme au Japon entre un marxisme en voie de naturalisation et un marxisme né en Russie. Yamakawa ou Fukumoto seraient ainsi l'un comme l'autre les représentants antagoniques de cette tentative de « japoniser » le marxisme ${ }^{22}$.

Le différend tactique et politique qui oppose Yamakawa aux fukumoto-istes en 1924-26 s'appuie sur une analyse du capitalisme japonais et de la structure politique de la classe dominante, c'est-à-dire sur l'idée que le développement de l'État japonais moderne possède des caractéristiques historiques particulières qui nécessitent la mise au point d'une stratégie politique particulière. Pour Yamakawa, le système japonais est « semiféodal » depuis la révolution Meiji : l'institution impériale, l'existence d'une noblesse d'État, la Constitution particulièrement peu démocratique reflètent les difficultés de la bourgeoisie capitaliste moderne japonaise à s'imposer sur la scène politique comme classe autonome. Mais les émeutes du riz ont entraîné, en 1918, la chute des factions féodales (l'influence des hommes issus des anciens clans de samouraïs du Sud-ouest) et les gouvernements qui se succèdent depuis sont bien des gouvernements bourgeois, sous tutelle bureaucratique. Yamakawa explique l'insuffisance démocratique et la violence de la répression contre les mouvements oppositionnels par l'inexistence, au Japon, d'une véritable révolution bourgeoise. Le mouvement ouvrier doit donc simultanément mettre un terme aux institutions féodales qui subsistent et achever la démocratisation complète de la société à la place de la bourgeoisie. Il doit donc prendre la tête d'un front uni de l'ensemble des couches exploitées et constituer ce parti prolétarien ouvert d'un type nouveau, un parti de masse (donc non léniniste) capable d'imposer ses objectifs à une bourgeoisie immature et incapable de s'arracher à la tutelle idéologique des institutions semi-féodales.

Par sa vision de l'histoire récente du Japon, Yamakawa impose à la gauche socialiste et communiste japonaise l'idée que la stratégie politique est liée à l'analyse historique que l'on propose du passé proche et du présent. Et, effectivement, une grande partie de l'énergie théorique des intellectuels marxistes japonais va se concentrer désormais sur l'analyse de l'histoire du capitalisme et de l'État au Japon. Ce qui est à l'origine une dispute au sein du parti prend cependant rapidement l'allure d'une polémique d'envergure nationale dans laquelle interviennent militants, mais aussi historiens et économistes professionnels, marxistes ou proches du marxisme, soit pour défendre les 
thèses officielles du parti, soit pour défendre les thèses dissidentes. Ces dernières se conçoivent comme le produit d'un marxisme non léniniste face à l'orthodoxie défendue par les théoriciens de l'école kôza.

Du coup, à la fin des années 1920 et au cours des années 1930, un certain nombre d'intellectuels marxistes s'engagent dans un travail d'analyse et de réflexion dont l'objet est la nature du développement de la société japonaise à travers le changement de ses structures sociales. Le travail part dans deux directions : une analyse qui porte sur les structures économiques et la nature de l'État; une autre dont le travail s'intéresse surtout à l'idéologie japonaise et aux tendances liées aux phénomènes culturels.

\section{L'école kôza} Japon moderne et il est analysé comme la mise en phce d' Ǵtat absolutiste considét comme «le stade suprême du féodalisme ». Le système économique est caractérisé par la cohabitation entre un secteur capitaliste et des formes archaïques de production. Vers 1900, le capitalisme prend le dessus, mais c'est pour évoluer immédiatement en système impérialiste à caractère autoritaire. Ce système est désigné comme «capitalisme militarisé et semi-féodal». L'ouvrage collectif propose une analyse suffisamment percutante et originale de la société japonaise pour qu'il devienne le mot éponyme d'un courant de pensée en tant que tel : «Leçons 》 ou «Cours », en japonais kôza, a en effet donné naissance, en jargon de l'époque, au mot qui désigne ce courant marxiste d'historiens et d'économistes proches du Parti communiste japonais, l'école kôza (kôza ha). C'est avec cette école kôza qu'on commence à désigner, en japonais courant, sous le nom de tennôsei (système impérial), le système de domination dictatoriale centré sur l'Empereur (tennô) système qui s'étend jusqu'à la sphère de la psychologie collective et qu'on n'avait jusque-là pas véritablement identifié comme tel. L'État se sentit, en tous cas, suffisamment mis en cause par cette analyse pourtant complexe et aride pour que l'ouvrage soit, peu après sa parution, saisi, censuré, puis interdit de publication, tandis que les auteurs étaient parfois arrêtés ${ }^{23}$.

Celui qui joua un rôle central dans la mise en place du projet kôza, c'est Noro Eirarô. Il fit partie du mouvement étudiant à l'université Keiô puis entra au Parti communiste, mais se lança surtout dans un travail théorique d'analyse de la féodalité japonaise, de la rénovation Meiji et du capitalisme qu'il publia seul, dès 1930, sous le titre Histoire $d u$ développement du capitalisme au Japon. Cet ouvrage, qui commence par l'histoire des débuts du capitalisme dans l'archipel et se termine par une analyse de la situation de l'époque, avance que le capitalisme japonais possède plusieurs caractéristiques. Pour Noro, «Meiji est évidemment une révolution politique, mais surtout une révolution sociale radicale. Ce n'est pas seulement une restauration impériale, mais c'est un changement social puissant qui a placé capitalistes et patrons en position de 
domination ${ }^{24}$. » Dans ces conditions, et sous l'influence des grandes puissances, le Japon a dû changer de cours et ce changement a contribué au processus d'incubation du capitalisme dans le cadre spécifique de l'archipel.

31 Malgré sa jeunesse et son inexpérience comme historien, Noro Eitarô avance des idées neuves. C'est lui qui, le premier, a cette idée que l'une des spécificités du capitalisme japonais réside dans la coexistence exacerbée de secteurs ultra-modernes et performants avec certains secteurs hyper-archaïques (l'agriculture, le bâtiment, etc.), non exposés à la concurrence internationale. Il est aussi l'un des premiers à montrer que l'histoire de Meiji reste à faire...

Noro Eitarô a eu l'idée des principes à l'origine de l'école kôza. Il rassemble des théoriciens, qu'ils soient ou non du parti, notamment ceux impliqués dans diverses sociétés de recherche et de réflexion sur le marxisme. Mais, du fait de l'aggravation de la tuberculose qu'il contracte en 1926 et de son activité militante débordante, il ne peut mener à bien complètement le travail. Victime aussi de la censure, il finit par se faire arrêter en 1932 et il meurt en prison, malade, brisé par les privations, en 1934.

L'école kôza cherchait à appliquer à l'étude de la société japonaise les principes du matérialisme historique et elle contribua à faire grandement progresser la recherche en histoire économique tout particulièrement. En 1934, l'un des contributeurs de la série, Yamada Moritarô publia à son tour une Analyse du capitalisme au Japon, qui se voulait un peu l'équivalent du livre de Lénine consacré au Développement du capitalisme en Russie (1899). La même année, Hirano Yoshitarô publia Les mécanismes du capitalisme au Japon. Ces deux ouvrages apparaissent eux aussi comme des «classiques » de l'école kôza et mettent en avant les caractères particuliers du capitalisme nippon dominé par ses caractères militaristes et « semi-féodaux ».

Deux autres historiens de ce courant jouèrent un rôle important. Tout d'abord, Hani Gorô (1901-1983), un historien de l'université de Tokyo très critique à l'égard de l'académisme japonais, lecteur de Croce et Labriola, mais aussi de Sorel. Ses analyses de Meiji mettent l'accent sur le rôle des paysans dans le processus de désagrégation de la société dans les années 1860, mais réévaluent aussi le rôle des pressions étrangères (Recherches sur l'histoire de la restauration Meiji). Hani Gorô n'est pas seulement historien. Il est aussi essayiste et il mène un combat antifasciste acharné. Pour éviter la censure, voire une arrestation, Hani Gorô déserte le combat politique et publie, en 1939, un ouvrage intitulé Michel-Ange, dans lequel il se livre à une analyse de la citoyenneté urbaine dans la « ville libre » qu'était la Florence de la Renaissance. Après-guerre, Hani Gorô deviendra le principal chef de file du courant kôza.

De son côté, Hattori Shisô (1901-1956) publie des travaux sur la fin de la période d'Edo et il est l'un des premiers historiens à insister sur le caractère déjà développé de l'économie sous les Tokugawa, qu'il qualifie d'« âge des manufactures ». Ses travaux sur la révolution Meiji et sur le mouvement pour la liberté et les droits du peuple concluent à l'échec final des réformes entreprises alors et consolident sa vision d'un État meijien conçu comme un moment d'absolutisme.

$\mathrm{Au}$ bout du compte, les apports de l'école kôza sont indéniables dans plusieurs domaines. D'abord, il existe un vocabulaire qui passe pour la première fois la rampe des jargons militants : contradictions, révolution, développement, lutte, capitalisme... Il ne s'agit pas non plus d'une pensée scholastique, comme on a pu la décrire par la suite. Par exemple, Hani Gorô est en désaccord avec Noro Eitarô quand ce dernier affirme que Meiji, c'est la mise en place d'un absolutisme qui maintient les rapports féodaux, 
notamment dans les campagnes. Hani Gorô parle plutôt, de son côté, de révolution bourgeoise inachevée. L'école kôza est une école précisément, c'est à dire un lieu vivant où débats et critiques se déroulent en toute liberté. Ces jeunes chercheurs passionnés ne sont pas engagés dans une dynamique de construction d'un savoir de nature totalitaire. Au bout du compte, les travaux de l'école kôza, au-delà de leur aspect daté et d'une écriture réalisée dans l'urgence, sont les premiers à prendre en compte en histoire les structures socio-économiques lourdes, à mettre en avant la combinaison de plusieurs systèmes économiques en parallèle, à insister sur la diversité interne de la société japonaise entre 1870 et 1930, et, enfin, à jouer sur les tendances universelles du capitalisme et les particularités de la société japonaise.

Mais, d'un autre côté, leurs travaux ne sont pas que de simples recherches. Ils ont aussi pour objectif de montrer quelles seraient les bases de la future révolution à venir. Pour l'école kôza, la révolution à venir ne pouvait qu'être une révolution bourgeoise, puisque le régime né avec Meiji autour de la figure "absolutiste» de l'empereur avait été incapable de s'atteler réellement aux nécessaires réformes de structure que la révolution à venir aurait à cœur de réaliser. De son côté, rendu prudent par l'échec de la révolution chinoise de 1927 à Shanghai, le Komintern revint à des thèses classiques et très euro-centriques sur "l'immaturité " des sociétés asiatiques en général et de la société japonaise en particulier : il en conclut à la nécessité d'une révolution au Japon, dans laquelle le PCJ devrait se faire le principal porteur des objectifs d'une véritable révolution bourgeoise. Les Thèses du Komintern de 1932 consacrées à la situation japonaise, dont il semblerait qu'elles furent rédigées par le communiste finlandais Kuusinen, alors à la tête du Bureau oriental de l'Internationale communiste, rejoignaient par certains aspects les propos de l'école kôza, qui se sentit ainsi soutenue par Moscou.

\section{L'école rônô}

Le courant kôza doit néanmoins affronter très rapidement des critiques de la part d'autres intellectuels marxistes qui défendent le point de vue selon lequel Meiji avait, au contraire, bien des aspects d'une révolution bourgeoise et donc que la révolution à venir serait bien une révolution prolétarienne. Pour ces derniers, les aspects archaïques du système japonais sont transitoires et voués à disparaître. Pour Yamakawa et ses amis, regroupés depuis 1927 dans la tendance rônô, du nom de la revue Rônô (Ouvriers et paysans) qu'ils publient, l'importance du capitalisme financier au Japon, la croissance des conglomérats industriels, les zaibatsu, l'expansion impérialiste, tout indique au contraire que le Japon est devenu un pays industriel avancé et que les tendances "semi-féodales" sont en voie de résorption. La société japonaise est une société qui a bel et bien fait le saut dans l'univers capitaliste, même si les formes politiques de l'État et l'idéologie qu'il distille sont encore empreintes de «traces féodales ».

Comme les membres de l'école kôza, ceux de rônô vont constituer un courant important de recherches et de travaux consacrés à l'histoire de l'économie et de l'État. Mais, à la différence des membres de l'école kôza, qui concentrent leurs recherches sur Meiji, les tenants de l'école rônô, eux, tentent d'analyser le capitalisme japonais dans ses derniers développements, depuis la révolution industrielle des années 1900 et l'émergence d'un puissant capitalisme depuis 1920. L'un des membres les plus brillants de cette école est 
Inomata Tsunao (1889-1942), un économiste qui enseignait à l'université Waseda. Inomata rédigea plusieurs ouvrages consacrés à l'impérialisme, au capitalisme monopoliste ou à la question agraire. Au début des années 1930, il se pencha sur la nature de la crise économique, qui s'aggravait notamment dans les campagnes, où la situation devint souvent dramatique. Cette situation est bien décrite dans l'un de ses ouvrages de l'époque, considéré aujourd'hui comme un classique ${ }^{25}$. Inomata se rendit en effet dans plusieurs départements du pays, écouta les doléances des paysans et décida "d'écrire en ayant encore leur témoignage vif en tête ". Inomata parvint à dresser un tableau non seulement des réalités mais aussi des mentalités paysannes en même temps qu'il prenait un point de vue plus large d'économiste.

Les membres de l'école rônô ne sont plus communistes, mais se disent marxistes. Yamakawa Hiroshi le proclame quand il explique que les Japonais n'ont pas à imiter la social-démocratie allemande ou le bolchévisme russe. Il faut revenir à Marx , écrit-t-il ${ }^{26}$. Ceux de la rônô -ha sont proches des partis prolétariens, non communistes et souvent non affiliés. Tous, d'ailleurs, ne publient pas dans la revue Rônô. On peut déceler chez eux une pointe de nationalisme quand ils refusent de se laisser contrôler par les idéologues du Komintern qui décident, depuis Moscou, de la ligne stratégique du mouvement communiste japonais. Ils refusent d'admettre que le Japon soit « un pays arriéré puisqu'asiatique et non occidental ", et donc que la stratégie politique à mettre en place soit subordonnée à cette vision d'une société encore semi-féodale partagée par les intellectuels de l'école kôza et le Komintern. Ils sont souvent très actifs dans ce qui se révèlera un échec : créer un Front Populaire en 1936. Ils tomberont victimes de la répression policière à leur tour en 1938, et surtout en 1940, et leur groupe est dispersé.

Il est à noter que le débat kôza-ha/rônô-ha qui fut vif dans les années 1930 et repartit de plus belle dans l'après-guerre, tourne en grande part autour de la nature de la révolution/rénovation Meiji, c'est-à-dire la transformation des structures étatiques lors des réformes entreprises, pour l'essentiel, entre 1868 et $1873^{27}$. Savoir s'il s'agit là ou non d'une révolution bourgeoise, telle qu'on l'entendait vers 1930, revient à poser implicitement la comparaison avec la Révolution française de 1789 et ce qui la caractérise fondamentalement. Si on part du principe que Meiji a détruit l'ancien régime des Tokugawa comme la Révolution a liquidé la monarchie absolutiste, alors on peut évoquer une révolution bourgeoise. Mais peut-il y avoir révolution bourgeoise sans intervention de la bourgeoisie et sans mouvement populaire de masse ? Faut-il considérer le processus ou son résultat? Les débats risquaient d'être stériles, d'autant que les études historiques sérieuses sur l'avant- et l'après-1868 restaient bien rares en ce temps. Certains, comme Hani Gorô, cherchèrent à montrer que la paysannerie japonaise était entrée en mouvement avant 1868 dans le cadre de mouvements de nature millénariste qui rappelaient certains épisodes de la Grande Peur de l'été 89, ce qui n'est certes pas faux. Mais les études historiques sérieuses manquaient pour analyser ce qui s'était passé dans le Japon soixante ans plus tôt. Ce débat, quelles qu'en soient les limites, eut une influence fondamentale sur la formation des sciences sociales au Japon. Ce fut précisément tout à la gloire de ce débat que d'avoir lancé une réflexion qui inspira par la suite toute une série de travaux historiques dont l'influence perdure encore ${ }^{28}$. 
Avant-guerre, le débat entre l'école kôza et l'école rônô agitait beaucoup les cercles intellectuels marxisants, notamment les historiens et les économistes. À l'origine, il s'agissait de débats qui concernaient surtout la frange politisée des intellectuels, mais, à partir du début des années trente, il déborda largement le cercle des initiés. Les grandes revues, comme Chûô kôron et Kaizô, s'en firent l'écho. Mais, à partir des années 1937-38, la répression se fit trop dure : limitation de la liberté d'expression, censure, intimidations, arrestations... Le débat resurgit de plus belle, après-guerre, quand il fallut faire l'analyse de la nature des réformes démocratiques imposées par l'armée d'occupation américaine. La plupart des membres du courant rônô rejoignirent, au lendemain de la guerre, l'aile gauche du parti socialiste - à commencer par Yamakawa Hitoshi lui-même - tandis que les membres du courant kôza restaient proches du PCJ.

Le débat sur la nature du capitalisme japonais opposant différentes interprétations issues des courants marxistes eut une influence suffisante pour structurer les représentations de la période moderne pendant près d'un demi-siècle. Jusque dans les années 1960-70, le marxisme dominait de fait l'idée que l'on se faisait de l'histoire au Japon, une histoire qui ne parvint pas à s'émanciper du paradigme attirant d'une modernité «tordue ", "gauchie», « inachevée ${ }^{29}$, censée expliquer la misère, l'inégalité sociale et l'irrationalité des décideurs politiques.

\section{Le « marxisme raffiné » de Tosaka Jun}

Cependant le débat intellectuel au sein du marxisme japonais dans les années 1930 ne saurait se réduire à celui opposant les deux écoles de pensée kôza et rônô. Le philosophe marxiste Tosaka Jun (1900-1945), par exemple, resta de son côté parfaitement imperméable à cette polémique : lui ne s'intéressait guère aux questions économiques et sa réflexion portait plutôt sur le champ culturel, sur l'idéologie nationale et sur l'importance des «superstructures ». Son travail a pu être considéré comme en phase avec ce qu'il est convenu d'appeler le marxisme occidental ${ }^{30}$, a d'ailleurs pu être comparé à celui de Gramsci ${ }^{31}$ et est empreint d'une forme de pessimisme: dans les années 1930, Tosaka ne croyait plus guère en la possibilité d'une révolution japonaise et l'histoire lui donna finalement raison sur ce point. Il écrivit son œuvre principale entre 1935 et 1938, dans un contexte politiquement de plus en plus difficile, et s'interrogeait sur le pourquoi de l'échec des forces révolutionnaires dans l'archipel.

Tosaka Jun fait partie de la mouvance de l'école philosophique de Kyôto et s'en détache pour fonder, en 1932, un groupe de recherche sur le matérialisme, qui publie une petite revue, Études matérialistes. En 1934, il est expulsé de l'université privée Hôsei de Tokyo, dans laquelle il enseignait, pour activités subversives. C'est alors qu'il publie ses principaux ouvrages, notamment l'Idéologie japonaise (1935) ${ }^{32}$. Arrêté en 1938, il est emprisonné et meurt affaibli et malade, dans la prison de Nagano le 9 août 1945, une semaine à peine avant la capitulation, dévoré par la gale sans jamais avoir été soigné. Son influence intellectuelle sur les jeunes générations sera capitale dans les années 1950-1960.

Tosaka rejette l'idée que l'économie détermine les superstructures. Pour lui, les facteurs économiques et la conscience relèvent de sphères qui peuvent s'influencer mutuellement mais qui ne sont pas reliées par une causalité mécanique. Elles gardent chacune leur autonomie. Pour Tosaka, les institutions, à savoir le système scolaire, les 
médias, l'activité publique quotidienne - la société civile en termes gramsciens imposent leurs normes sur les gens, disent aux gens comment penser et agir, et font en sorte de produire de l'adhésion collective au système, au point de laisser croire aux gens ordinaires qu'ils pensent selon leur propre volonté. Cette internalisation des normes sociales est fondamentale et plus efficace que les moyens de répression habituels (police, justice, armée...).

En 1936, dans un ouvrage consacré à La pensée et la coutume, Tosaka examine les mœurs et la vie quotidienne, qu'il analyse comme les éléments centraux conduisant à cette internalisation des normes chez les individus. Les coutumes populaires, dit-il, ne peuvent être saisies par les concepts marxistes orthodoxes reliant les superstructures aux bases économiques d'une société. Or, c'est par le biais de ces coutumes présentées comme des normes évidentes que s'exerce la domination. En se conformant aux coutumes, on pense agir d'une manière moralement correcte. Les masses populaires adoptent les normes socialement dominantes, non parce qu'elles n'ont aucune conscience de classe, mais parce que l'adhésion à ces normes légitime leur action au quotidien et produit une morale qui leur convient. La société fonctionne parce que les masses donnent leur consentement à l'idéologie dominante. Pour Tosaka - comme pour Gramsci - , il faut intervenir sur la manière de penser et d'agir des couches populaires plutôt que de lancer des actions révolutionnaires contre l'État, et donc imaginer des stratégies de conquête du pouvoir sur le long terme par une action quotidienne démontant - via les médias par exemple (Tosaka dit « les journalistes ») - le caractère aliénant de la " pensée ordinaire ».

Maruyama Masao (1914-1996), le grand philosophe des idées de l'après-guerre, écrivait, en 1961, que ceux qui ont mis en pièce les manières traditionnelles de pensée au Japon, ce sont les chrétiens à l'ère Meiji et les marxistes à l'ère Taishô (1912-1926) ${ }^{33}$. Ces deux courants «se sont dressés en fin de compte de manière frontale " contre les systèmes de pensée dominants et ont eu pour destin « de jouer un rôle commun dans l'histoire de la pensée en s'intégrant dans le paysage intellectuel japonais ». Ils se sont adaptés au climat intellectuel qui régnait dans ce pays et ils y ont accompagné une révolution intellectuelle. Non seulement ces courants ont développé une critique radicale, mais, en ce qui concerne le marxisme, Maruyama écrivait qu'il s'agissait, " pour la première fois au Japon, d'une méthode générale capable d'intégrer les réalités sociales, la politique, le droit, la philosophie et l'économie, qui jusqu'alors avaient toujours été pensés de manière indépendante ${ }^{34} »$. Mais il ajoutait que, en même temps que le marxisme réunifiait les sciences sociales, il fabriquait son propre poison autodestructeur en devenant une "religion de la théorie ", présentant une pensée souvent trop figée. C'est néanmoins tout à la gloire du marxisme japonais d'avant-guerre que d'avoir été en mesure de produire une pensée critique. 


\section{NOTES}

1. Christine LÉVY, «Kôtoku Shûsui et l'anarchisme », Ebisu, Études japonaises, $n^{\circ} 28$, printemps-été 2002

2. Ко̂тоКU Shûsui, L'impérialisme, le spectre du XXe siècle, CNRS Éditions, 2008.

3. Ітон Makoto, La crise mondiale, Théorie et pratique, EDI, Paris, 1987, p. 16.

4. Pierre François souYRI, "Les émeutes du riz de 1918: le grand tournant ", Ebisu, Études japonaises, $\mathrm{n}^{\circ}$ 53, 2016.

5. Le Japon est le premier pays où fut traduit intégralement Le Capital. Voir ITOH Makoto, La crise mondiale, Théorie et pratique, op. cit., p.5. Takabatake évoluera plus tard sur des positions discutables, faisant de l'État l'élément central des nécessaires réformes pour détruire le système sur lequel doivent s'appuyer les forces de contestation, et flirtant avec l'extrême droite nationaliste.

6. Peter DUUS et Irwin SCHEINER, «Socialim, Liberalism and Marxism », dans Bob T. Wakabayashi (dir.), Modern Japanese Thought, Cambridge University Press, 1998, p.183.

7. Né en 1880 , fils d'un paysan aisé de la région d'Okayama, Yamakawa Hitoshi interrompt ses études à l'école Dôshisha de Kyôto en 1897. Arrêté en 1901 pour avoir signé un article expliquant que le mariage du prince impérial était « un événement funeste ", il purge trois ans de prison à Tôkyô. Il adhère au parti socialiste en 1906 et est proche de Kôtoku Shûsui Arrêté en 1908, il est condamné de nouveau à deux années de prison et échappe ainsi aux arrestations de 1910 dans le cadre de l'affaire de lèse-majesté. Pendant "l'ère d'hiver ", il reprend en province un commerce de pharmacie, mais regagne Tôkyô en 1916 pour rejoindre le groupe de Sakai Toshihiko et épouser la féministe Yamakawa Kikue. Le couple s'impose peu après sur le devant de la scène intellectuelle, Hitoshi par ses articles critiques contre les démocrates, tandis que Kikue fait de même par ses interventions dans le débat sur la maternité et ses positions sur l'autonomie du féminisme au sein du mouvement socialiste.

8. YAMAKAWA Hitoshi, "Musankaikyû undô no hôkôtenkan" (Changement de cap pour le mouvement prolétarien), Zen'ei (Avant-garde), juillet-août 1922, repris dans Shakaishugi (Le socialisme), tome 15 de Gendai Nihon shisô taikei (Grande série sur la pensée japonaise contemporaine), Chikuma shobô, 1963, ré-éd. 1975.

9. Kaizô (en japonais, réforme, reconstruction) est le nom donné à une revue fondée en 1919 (elle disparait en 1955). Kaizô est l'un des mots fétiches de la période qui s'ouvre vers 1920 autour de l'idée d'une reconfiguration du système politique et social japonais. La revue se caractérise par des numéros spéciaux : «La critique socialiste de la question du travail» (juillet 1919), « Maîtriser le capitalisme » (août 1919), «La lutte des classes» (décembre 1919). Elle donne la parole sans restriction à des anarchistes, des marxistes ou des chrétiens et intervient dans la plupart des grands débats de l'époque, au point de devenir la revue de référence de son temps jusqu'au milieu des années 1930, où elle devient la cible de l'extrême-droite et est à plusieurs reprises censurée. Kaizô est finalement interdite en 1944 et reparaît en 1946. La maison d'édition à l'origine de la revue fut à l'initiative de l'invitation au Japon, dans les années 1920, de grandes figures intellectuelles, à commencer par Albert Einstein ou la féministe américaine Margaret Sanger. Elle lance la première collection japonaise de livre de poche en 1926. Elle publie, à partir de 1932, en trente-cinq volumes, les premières Euvres complètes de Marx et Engels dans le monde. Voir. KANo Masanao, Taishô demokurashii no shisô to bunka (Pensée et culture de la démocratie de l'ère Taishô), Iwanami kôza Nihon shi, tome 18, Iwanami shoten, 1975. Voir également KUROKAWA Midori, Kaizô no jidai (L'époque de Kaizô), Iwanami kôza Nihonrekishi, tome 17, Iwanami shoten, 2014, p. 173-208. 
10. Le groupe s'étant constitué dans la clandestinité la plus totale, la documentation, y compris de source policière, est quasi inexistante. Seuls subsistent des témoignages a posteriori qui, parfois, divergent.

11. Depuis 1900, les lois répressives visant à étouffer les syndicats et les mouvements sociaux permettent de maintenir sous tension policière les militants. La répression se raidit d'autant contre les communistes et apparentés que le gouvernement japonais est en guerre officielle avec le régime bolchévik russe depuis l'intervention en Sibérie (1918-1922). Pour les dirigeants japonais, l'ancien empire des tsars devenu URSS fait peser une menace sur les frontières septentrionales du Japon. Au Japon, les communistes sont alors considérés comme des agitateurs, qui plus est au service d'une grande puissance, donc doublement subversifs.

12. Sur la répression cette année-là, voir KOBAYASHI Takiji, Le 15 mars 1928, [publié en 1929], traduit par Mathieu Capel, Éditions Amsterdam, 2020.

13. Sur les origines du mouvement socialiste japonais, voir Pierre François souYRI, Moderne sans être occidental, aux origines du Japon d'aujourd'hui, 2016, Gallimard, p. 410-441.

14. Au lendemain du Grand Séisme du Kantô ( $1^{\mathrm{er}}$ septembre 1923), qui fit plus de cent mille morts dans l'agglomération de Tokyo, de nombreux militants furent arrêtés. Le leader anarchiste Ôsugi Sakae fut alors torturé et étranglé dans un commissariat de Tokyo, ainsi que sa compagne, l'anarcho-féministe Itô Noe, et son jeune neveu, âgé de six ans [sic!], qui furent l'un comme l'autre étranglés dans une geôle du commissariat. Le lieutenant Amakasu Masahiko fut condamné pour ce triple assassinat à dix ans de prison et libéré en 1926. Devenu l'un des hauts responsables de l'administration de la Mandchourie pendant la guerre, il se suicida lors de la défaite en août 1945.

15. Des tendances anarcho-syndicalistes émergent vers 1925 dans les syndicats paysans regroupant petits fermiers et prolétaires des campagnes, reflets d'une agitation croissante dans la paysannerie.

16. Par parias, nous entendons ici ces catégories de la population qui, à l'époque d'Edo, étaient regroupées dans des ghettos urbains ou ruraux dont elles ne pouvaient guère sortir et qui se livraient à diverses occupations professionnelles frappées d'interdits : les bouchers, les tanneurs, les teinturiers, les cordonniers (connus sous le nom générique d'eta, les « souillés »), les acteurs de théâtre, les saltimbanques, voire certaines prostituées ou criminels (désignés souvent comme hinin, les «non-humains). Dans les années 1870, ces gens sont regroupés dans des hameaux (buraku) Vers 1918-1920, les descendants des parias commencent à renverser le discours dominant, affirmer leur identité et prendre leur sort en main. Ils créent un mouvement fondé sur l'autonomie de leurs revendications et adoptent pour se désigner avec fierté la nouvelle appellation officielle de burakumin (gens des hameaux). Ils représentent, au début du $\mathrm{xx}^{\mathrm{e}}$ siècle, environ 1,5\% de la population du Japon. Ils créent finalement en 1922 une association politique à qui ils donnent le nom de Suiheisha (la Société horizontale), très influencée par les idées radicales, qui adopte comme symbole le drapeau noir avec une double couronne d'épines rouges en son centre.

17. La traduction de morceaux choisis du Capital par Kawakami-Miyagawa publiée en 1927 a été vendue à plus de 300000 exemplaires en 1935 ! Toutes les éditions cumulées du Capital en langue japonaise ont été vendues à plus de 1500000 exemplaires en 1950... et Le Capital financier de R. Hilferding (1910) est publié en japonais en 1927. Voir Ітон Makoto, La crise mondiale, Théorie et pratique, op.cit, p.19.

18. YASUDA Tsuneo, "Marukusushugi to chishikijin » (Le marxisme et les intellectuels), Nihon tsushi 18, 3, Iwanami shoten, 1994. p.207-237.

19. Gavin Walker, Guide de lecture, le marxisme au Japon. http://revueperiode.net/guide-de-lecturele-marxisme-au-Japon/

20. Paul F. LANGER, Communism in Japan, A Case of political Naturalization, Stanford University Press, 1972, p. 10. Fukumoto est d'ailleurs arrêté par la police l'année suivante lors des grandes rafles 
anticommunistes de 1928 et condamné, plus tard, à dix ans de prison, qu'il purge dans le Hokkaidô (il est libéré en 1942).

21. Ibid., p. 5.

22. Germaine A. Hoston, Marxism and the Crisis of Development in Prewar Japan, Princeton University Press, 1986, p.41.

23. Dans le Japon des années noires, les opposants sont condamnés, parfois lourdement, mais la justice japonaise ne condamne pas un seul opposant à la peine de mort. Les militants qui meurent en prison sont victimes de «bavures" policières - à vrai dire assez systématiques - ou de manque de soins, mais il n'y a pas d'exécutions « légales ». Nombre de militants arrêtés lors des rafles de 1928 ou de 1932-33 et qui ont écopé de dix ou quinze ans de réclusion sortent de prison après avoir purgé leur peine, en pleine guerre, vers 1942-43, C'est le cas, on l'a vu plus haut, de Fukumoto Kazuo. On estime à 70000 le nombre de ceux qui furent arrêtés pendant la période 1928-1945. On reste loin des 500000 arrestations en Allemagne pendant la période qui suit la prise du pouvoir par les Nazis en 1933.

24. Noro Eitarô, "Nihon shihonshugi hattatsushi », repris dans Marukushizumu (Le marxisme) tome II, tome 21 de Gendai Nihon shisô taikei, Chikuma shobô, 1966, p. 82. Édition de poche chez Iwanami bunko en 1983.

25. INOMATA Tsunao, Tôsa hôkoku - Kyûbô no nôson (Les Villages de la Misère, rapport d'enquête sur le terrain), 1934. Réédité en poche aux éditions Iwanami bunko en 1982.

26. Cité par Germaine hoston, Marxism and the Crisis of Development..., op. cit. p. 40.

27. Sur ce point, voir Pierre François souYRI, Moderne sans être occidental..., op. cit.

28. NARITA Ryûichi, Sengo shi nyûmon (Une introduction à l'histoire dans l'après-guerre), Kawade shobô, 2001. Voir également sugIHARA Kaoru dans Actuel Marx, $\mathrm{n}^{\circ} 2$, le Marxisme au Japon, L'Harmattan, 1987, p.32.

29. Pierre-François SOUYRI, «La modernité japonaise dans tous ses états », dans Le Débat, $\mathrm{n}^{\circ} 153$, janvier-février 2009, p.88-99.

30. Perry ANDERSON, Considerations on Western Marxism, London, NLB, 1976.

31. TAKAHIRO Chino, «Is Western Marxism Western? The Cases of Gramsci and Tosaka », Journal of World Philosophies 2 (summer 2017), p. 28-41.

32. Des morceaux choisis de Tosaka Jun ont été traduits en anglais et en allemand mais rien à ce jour en français. TOSAKA Jun, Tosaka Jun, A Critical Reader, édité par Ken Kawashima, Fabian Schäfer et Robert Stolz, New York, Cornell University, 2013.

33. MARUYAMA Masao, Nihon no shisô (La pensée japonaise), Iwanami shinsho, 1961, p. 15.

34. MARUYAMA Masao, Ibid., p. 55.

\section{RÉSUMÉS}

Dans les années 1920 et 1930, le marxisme prend racine au Japon avant d'être contraint au silence par la répression. Il produit en ce temps une pensée nouvelle, « moderne » et ouverte, à l'origine de nombreux débats qui traversent la société dans son ensemble. La question de la nature spécifique du capitalisme japonais et donc de la société issue de la révolution de Meiji(1868-1873) devient centrale pour comprendre la nature de la future révolution. En creux, se profile le débat sur la nature « bourgeoise » ou non du mouvement qui met fin au régime féodal des Tokugawa avec un impensé qui est constant : celui de la comparaison avec la Révolution française de 1789. 
L'article passe en revue les différents apports du marxisme japonais aux sciences sociales et à la compréhension de la société contemporaine.

In the 1920s and 1930s, Marxism sets roots in Japan, before it is reduced to silence by oppression. During that time, it becomes the source of a new, "modern" and open school of thought that sparks numerous debates across the whole of the Japanese society. The issue of the peculiar nature of Japan's capitalism and, as such, of the society established by the Meiji Revolution (1868-1873), has become central to understand the nature of the future revolution. Underneath that looms the debate about whether the movement that led to the end of the Tokugawa's feudal regime was of a "bourgeois" nature or not, along with a constant, but silent, issue: comparing it with the French Revolution of 1789. This article will analyse the various inputs from Japan's Marxism to social sciences and to the understanding of the society of that time.

\section{INDEX}

Mots-clés : communisme, marxisme, Particularités du capitalisme japonais, Meiji et révolution française

Keywords : Communism, Marxism, Peculiar aspects of Japan's capitalism, Meiji and the French Revolution

\section{AUTEUR}

\section{PIERRE-FRANÇOIS SOUYRI}

Université de Genève 